\title{
Numerical solutions of doubly perturbed stochastic delay differential equations driven by Lèvy process
}

Received: 6 May 2011 / Accepted: 3 October 2011 / Published online: 11 April 2012

(C) The Author(s) 2012. This article is published with open access at Springerlink.com

\begin{abstract}
In this paper, the numerical solutions of doubly perturbed stochastic delay differential equations driven by Lèvy process are investigated. Using the Euler-Maruyama method, we define the numerical solutions, and show that the numerical solutions converge to the true solutions under the local Lipschitz condition. As a corollary, we give the order of convergence under the global Lipschtiz condition.
\end{abstract}

Mathematics Subject Classification (2010) 60H10 - 65C50 - 60J75

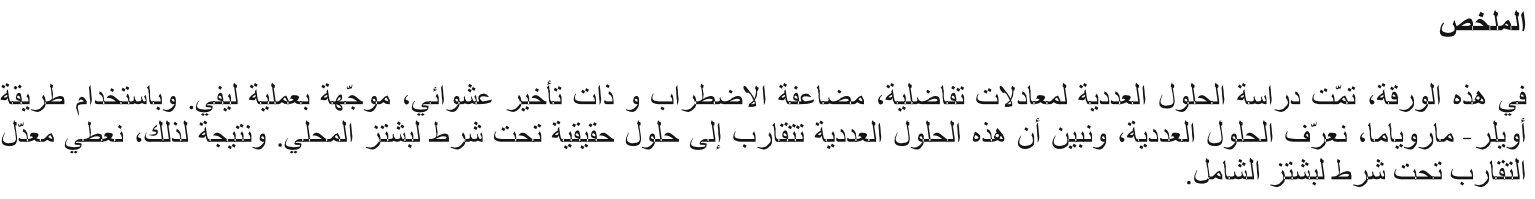

\section{Introduction}

The purpose of this paper is to study the numerical solutions of doubly perturbed stochastic delay differential equations (DPSDDEs) driven by Lèvy process. Such DPSDDEs take the form:

$$
\begin{aligned}
x(t)= & x(0)+\int_{0}^{t} f(x(s), x(\delta(s))) d s+\int_{0}^{t} g(x(s), x(\delta(s))) d B(s) \\
& +\int_{0}^{t} \int_{|l|<c} H(x(s-), x(\delta(s)-), l) \tilde{N}(d s, d l)+\alpha \sup _{0 \leq s \leq t} x(s)+\beta \inf _{0 \leq s \leq t} x(s),
\end{aligned}
$$

$\mathrm{X} . \mathrm{Wu}(\varangle)$

Department of Mathematics, Anhui polytechnic University,

8 East Zheshan Rd., Wuhu,

241000 Anhui, People's Republic of China

E-mail: aaxtwu@gmail.com

$\mathrm{X} . \mathrm{Wu} \cdot \mathrm{L}$. Yan

Department of Mathematics, Donghua University,

2999 North Renmin Rd., Songjiang,

Shanghai 201620, People's Republic of China

E-mail: litanyan@dhu.edu.cn 
where $0<\alpha, \beta<1$ and $\alpha+\beta<1 ; B(t), t \geq 0$ is a standard Brownian motion and $\widetilde{N}(d t, d l)$ is a compensated Poisson random measure; the mappings $f: \mathbb{R}^{d} \times \mathbb{R}^{d} \rightarrow \mathbb{R}^{d}, g: \mathbb{R}^{d} \times \mathbb{R}^{d} \rightarrow \mathbb{R}^{d \times m}$ and $H: \mathbb{R}^{d} \times \mathbb{R}^{d} \times \mathbb{R}^{d} \rightarrow \mathbb{R}^{d}$ are all Borel-measurable functions; $c \in(0,+\infty]$ is the maximum allowable jump size; $\delta(s)$ stands for the time delay.

The perturbed Brownian motion

$$
x(t)=B(t)+\alpha \sup _{0 \leq s \leq t} x(s)+\beta \inf _{0 \leq s \leq t} x(s),
$$

is a limit process from a "weak" polymers model of Norris et al. [14], and it also arises as the scaling limit of some self-interacting random walks (see e.g. $[17,18]$ ). This process behaves exactly as a Brownian motion except when it hit its past maximum (or minimum), where it gets an extra 'push'.

During the past 30 years, there are numerous papers concerned with Brownian motions perturbed at their extrema (see e.g. [2,4-6,15]). For example, Doney and Zhang [6] obtained the existence and uniqueness of the solutions for the following perturbed nonlinear diffusion process

$$
x(t)=x(0)+\int_{0}^{t} f(s, x(s)) d s+\int_{0}^{t} g(s, x(s)) d B(s)+\alpha \sup _{0 \leq s \leq t} x(s),
$$

where $f$ and $g$ are Lipschitz continuous functions. Recently, the large deviation principle for Eq. (1.2) was established by Bo and Zhang [2].

Most of DPSDDEs do not have explicit solutions and hence require numerical solutions. However, there are few numerical methods available for DPSDDEs yet. The numerical methods for stochastic differential equations (SDEs) have been well studied (see e.g. [7,12,16]), but this is not the case for stochastic delay differential equations (SDDEs) as it has been pointed out in [8]. Buckwar [3] studied the numerical solution of SDDEs under the global Lipschitz condition, and Mao [13] investigated it under the local Lipschitz condition. Moreover, some results of the numerical solutions of SDEs with jumps were obtained. One can see Li and Chang [10], Wang and Gan [19].

However, few work has been done on the numerical solutions of DPSDDEs. In this paper, we define the numerical solutions by the Euler-Maruyama method, and show that the numerical solutions converge to the true solution under the local Lipschitz condition. As a corollary, we give the order of convergence under the global Lipschitz condition.

The paper is organized as follows. In Sect. 2, we introduce some necessary notations and define the EulerMaruyama approximate solution to DPSDDEs. In Sect. 3, a number of useful lemmas are presented. In Sect. 4, the convergence of numerical solutions is proved in the sense of mean square. Finally, in Sect. 5, conclusions and discussions on future research topics are given.

\section{Preliminary notation and Euler-Maruyama method}

Throughout this paper, we let $(\Omega, \mathcal{F}, P)$ be a complete probability space with some filtration $\left\{\mathcal{F}_{t}\right\}_{t \geqslant 0}$ satisfying the usual conditions (i.e., the filtration is increasing and right continuous while $\mathcal{F}_{0}$ contains all $P$-null sets). Let $|x|$ be the Euclidean norm of $x \in \mathbb{R}^{d}$. Assume $\tau>0$ and $\mathbb{R}^{+}=[0,+\infty)$. Let $C\left([-\tau, 0] ; \mathbb{R}^{d}\right)$ be the family of continuous functions from $[-\tau, 0]$ to $\mathbb{R}^{d}$ with norm $\|\varphi\|=\sup _{-\tau \leq \theta \leq 0} \varphi(\theta)$. Denote by $L_{\mathcal{F}_{t}}^{p}\left([-\tau, 0] ; \mathbb{R}^{d}\right)$ the family of $\mathcal{F}_{t}$-measurable, $C\left([-\tau, 0] ; \mathbb{R}^{d}\right)$-valued random variables $\xi=\{\xi(s),-\tau \leq s \leq 0\}$ such that $E\|\xi\|^{p}=\sup _{-\tau \leq s \leq 0} E|\xi(s)|^{p}<+\infty$. Let $C(a)$ denote a constant, whose value depends only on $a$. For simplicity, we denote by $a \vee b=\max \{a, b\}$ and $a \wedge b=\min \{a, b\}$.

Let $B=(B(t), t \geq 0)$ be an $m$-dimensional standard $\mathcal{F}_{t}$-adapted Brownian motion and $N$ be an independent $\mathcal{F}_{t}$-adapted Poisson random measure defined on $\mathbb{R}^{+} \times\left(\mathbb{R}^{d}-\{0\}\right)$ with compensator $\widetilde{N}$ and intensity measure $v$, where $v$ is a Lèvy measure so that $\widetilde{N}(d t, d y):=N(d t, d y)-v(d y) d t$ and $\int_{\mathbb{R}^{d}-\{0\}}\left(|y|^{2} \wedge 1\right) v(d y)<\infty$. For Eq. (1.1), the initial data $x(0)=\xi(0) \in L_{\mathcal{F}_{t}}^{p}\left([-\tau, 0] ; \mathbb{R}^{d}\right)$, and $\delta:[0, \infty) \rightarrow \mathbb{R}$ is a Lipschitz continuous function which satisfies

$$
-\tau \leq \delta(t) \leq t \text { and }|\delta(t)-\delta(s)| \leq \rho|t-s|, \forall t, s \geq 0,
$$

for some positive constant $\rho$. 
In this paper, we make the following assumptions:

(H1) There exists a constant $K_{1}>0$ such that for all $-\tau \leq s<t \leq 0$

$$
E|\xi(t)-\xi(s)|^{2} \leq K_{1}(t-s) .
$$

(H2) For each $n$ and each $2 \leq \eta \leq p$, there exists a positive constant $K_{2}(n)$, such that

$$
\left|f\left(x_{1}, y_{1}\right)-f\left(x_{2}, y_{2}\right)\right|^{2} \vee\left|g\left(x_{1}, y_{1}\right)-g\left(x_{2}, y_{2}\right)\right|^{2} \leq K_{2}(n)\left(\left|x_{1}-x_{2}\right|^{2}+\left|y_{1}-y_{2}\right|^{2}\right)
$$

and

$$
\int_{|l|<c}\left|H\left(x_{1}, y_{1}, l\right)-H\left(x_{2}, y_{2}, l\right)\right|^{\eta} v(d l) \leq K_{2}(n)\left(\left|x_{1}-x_{2}\right|^{\eta}+\left|y_{1}-y_{2}\right|^{\eta}\right),
$$

for $x_{k}, y_{k} \in \mathbb{R}^{d}$ with $\left|x_{k}\right| \vee\left|y_{k}\right| \leq n, k=1,2$.

(H3) For $2 \leq \eta \leq p$, there exists a positive constant $K_{3}$ such that

$$
|f(x, y)|^{2} \vee|g(x, y)|^{2} \leq K_{3}\left(1+|x|^{2}+|y|^{2}\right)
$$

and

$$
\int_{|l|<c}|H(x, y, l)|^{\eta} v(d l) \leq K_{3}\left(1+|x|^{\eta}+|y|^{\eta}\right) .
$$

The existence and uniqueness of the solution for Eq. (1.1) can be guaranteed by (H2-H3) (see [11]). Now, we will define the Euler-Maruyama approximate solution of the DPSDDEs (1.1). Let the time-step size $\Delta \in(0,1)$ be a fraction of $\tau$, that is $\Delta=\frac{\tau}{N}$ for some sufficiently large integer $N$. Then the discrete Euler-Maruyama approximate solution is defined by

$$
\begin{aligned}
\bar{y}((k+1) \Delta)= & \bar{y}(k \Delta)+f\left(\bar{y}(k \Delta), \bar{y}\left(I_{\Delta}[\delta(k \Delta)] \Delta\right)\right) \Delta+g\left(\bar{y}(k \Delta), \bar{y}\left(I_{\Delta}[\delta(k \Delta)] \Delta\right)\right) \Delta B_{k} \\
& +\int_{|l|<c} H\left(\bar{y}(k \Delta), \bar{y}\left(I_{\Delta}[\delta(k \Delta)] \Delta\right), l\right) \widetilde{N}(\Delta, d l)+\alpha \sup _{0 \leq j \leq(k+1)} \bar{y}(j \Delta)+\beta \inf _{0 \leq j \leq(k+1)} \bar{y}(j \Delta) .
\end{aligned}
$$

with $\bar{y}(t)=\xi(t)$ on $-\tau \leq t \leq 0$. Here $I_{\Delta}[u]$ denotes the integer part of the real number $\frac{u}{\Delta}$. Thus, $I_{\Delta}[\delta(k \Delta)]$ represents the integer part of $\frac{\delta(k \Delta)}{\Delta}$. Clearly,

$$
-\tau \leq I_{\Delta}[\delta(k \Delta)] \Delta \leq k \Delta \text { for every } k \geq 0 .
$$

To define the continuous extension, let us introduce two step processes

$$
\begin{aligned}
& z_{1}(t)=\sum_{k=0}^{\infty} I_{[k \Delta,(k+1) \Delta)}(t) \bar{y}(k \Delta) \\
& z_{2}(t)=\sum_{k=0}^{\infty} I_{[k \Delta,(k+1) \Delta)}(t) \bar{y}\left(I_{\Delta}[\delta(k \Delta)] \Delta\right) .
\end{aligned}
$$

We define the continuous Euler-Maruyama approximate solution as follows

$$
\begin{aligned}
y(t)= & \xi(0)+\int_{0}^{t} f\left(z_{1}(s), z_{2}(s)\right) d s+\int_{0}^{t} g\left(z_{1}(s), z_{2}(s)\right) d B(s)+\int_{0}^{t} \int_{|l|<c} H\left(z_{1}(s), z_{2}(s), l\right) \widetilde{N}(d s, d l) \\
& +\alpha \sup _{0 \leq s \leq t} y_{1}(s, t)+\beta \inf _{0 \leq s \leq t} y(s)
\end{aligned}
$$


where

$$
y_{1}(s, t)=\sum_{j=0}^{I_{\Delta}[t]} I_{[j \Delta,(j+1) \Delta)}(s) \bar{y}(j \Delta)+I_{\left(I_{\Delta}[t] \Delta, t\right)}(s) y(s) .
$$

Let $y(t)=\xi(t)$, when $-\tau \leq t \leq 0$. Hence, for every $k \geq 0$ and $k \Delta \leq t<(k+1) \Delta$, we have

$$
\begin{aligned}
y(t)= & y(k \Delta)+\int_{k \Delta}^{t} f\left(z_{1}(s), z_{2}(s)\right) d s+\int_{k \Delta}^{t} g\left(z_{1}(s), z_{2}(s)\right) d B(s)+\int_{k \Delta|l|<c}^{t} \int_{0 \leq s \leq t} H\left(z_{1}(s), z_{2}(s), l\right) \tilde{N}(d s, d l) \\
& +\alpha \sup _{0 \leq s} y_{1}(s, t)+\beta \inf _{0 \leq s \leq t} y(s)-\alpha \sup _{0 \leq j \leq k} \bar{y}(j \Delta)-\beta \inf _{0 \leq j \leq k} \bar{y}(j \Delta) .
\end{aligned}
$$

Remark 2.1 We evaluate the jump integral by $\int_{|l|<c} H\left(\bar{y}(k \Delta), \bar{y}\left(I_{\Delta}[\delta(k \Delta)] \Delta\right), l\right) \widetilde{N}(\Delta, d l)$, where $\widetilde{N}(\Delta, d y)$ $=N(\Delta, d y)-v(d y) \Delta$. In Theorem 4.1, we shall show that the error of Euler-Maruyama approximate solution converges to zero in $L^{2}$ as $\Delta \rightarrow 0$.

\section{Lemmas and corollary}

The key contribution of this paper is to show that the Euler-Maruyama approximate solutions will converge to the true solutions of Eq. (1.1) under the local Lipschitz condition. The proof of the result is rather technical, so we present several lemmas before the main result.

Lemma 3.1 Assume that Eq. (2.4) holds. Then there exists a constant $C_{p, T}>0$, such that for $p \geq 2$,

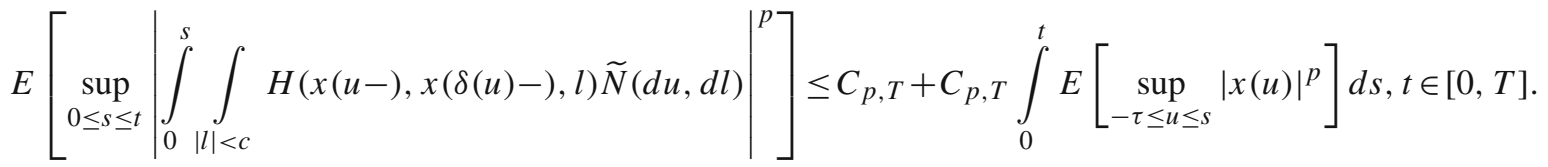

Proof By Theorem 2.11 in [9] (or Theorem 2.4 in [1]), there exists a positive constant $C(p)$, such that

$$
\begin{aligned}
& E\left[\sup _{0 \leq s \leq t}\left|\int_{0}^{s} \int_{|l|<c} H(x(u-), x(\delta(u)-), l) \tilde{N}(d u, d l)\right|^{p}\right] \\
& \leq C(p)\left[E \int_{0}^{t} \int_{|l|<c}|H(x(s-), x(\delta(s)-), l)|^{p} v(d l) d s+E\left(\int_{0}^{t} \int_{|l|<c} \mid H\left(x(s-),\left.x(\delta(s-), l)\right|^{2} v(d l) d s\right)^{\frac{p}{2}}\right] .\right.
\end{aligned}
$$

In view of Eq. (2.4), we obtain

$$
\begin{aligned}
& C(p)\left[E \int_{0}^{t} \int_{|l|<c}|H(x(s-), x(\delta(s)-), l)|^{p} v(d l) d s+E\left(\int_{0}^{t} \int_{|l|<c}|H(x(s-), x(\delta(s)-), l)|^{2} v(d l) d s\right)^{\frac{p}{2}}\right] \\
& \leq C(p)\left\{K_{3} E \int_{0}^{t}\left[1+|x(s-)|^{p}+|x(\delta(s)-)|^{p}\right] d s+T^{\frac{p}{2}-1} K_{3}^{\frac{p}{2}} E \int_{0}^{t}\left[1+|x(s-)|^{2}+|x(\delta(s)-)|^{2}\right]^{\frac{p}{2}} d s\right\}
\end{aligned}
$$




$$
\begin{aligned}
& \leq C(p) K_{3} T+2 C(p) K_{3} \int_{0}^{t} E\left[\sup _{-\tau \leq u \leq s}|x(u)|^{p}\right] d s+2^{\frac{p-2}{2}} K_{3}^{\frac{p}{2}} T^{\frac{p}{2}}+2^{p-1} K_{3}^{\frac{p}{2}} T^{\frac{p}{2}-1} \int_{0}^{t} E\left[\sup _{-\tau \leq u \leq s}|x(u)|^{p}\right] d s \\
& \leq C_{p, T}+C_{p, T} \int_{0}^{t} E\left[\sup _{-\tau \leq u \leq s}|x(u)|^{p}\right] d s,
\end{aligned}
$$

where $C_{p, T}=\left[C(p) K_{3} T+2^{\frac{p-2}{2}} K_{3}^{\frac{p}{2}} T^{\frac{p}{2}}\right] \vee\left[2 C(p) K_{3}+2^{p-1} K_{3}^{\frac{p}{2}} T^{\frac{p}{2}-1}\right]$. This completes the proof.

Lemma 3.2 Under condition (H3), for every $p \geq 2$, there exists a positive constant $C_{1}$, such that

$$
E\left(\sup _{-\tau \leq s \leq T}|x(s)|^{p}\right) \leq C_{1} .
$$

Proof For any $0 \leq s \leq t \leq T$, we get from Eq. (1.1),

$$
\begin{aligned}
\sup _{0 \leq s \leq t}|x(s)| \leq & \sup _{0 \leq s \leq t} \mid \xi(0)+\int_{0}^{s} f(x(u), x(\delta(u))) d u+\int_{0}^{s} g(x(u), x(\delta(u))) d B(u) \\
& +\int_{0}^{s} \int_{|l|<c} H(x(u-), x(\delta(u)-), l) \widetilde{N}(d u, d l)\left|+(\alpha+\beta) \sup _{0 \leq s \leq t}\right| x(s) \mid,
\end{aligned}
$$

which yields

$$
\begin{aligned}
\sup _{0 \leq s \leq t}|x(s)| \leq & \sup _{0 \leq s \leq t} \frac{1}{1-\alpha-\beta} \mid \xi(0)+\int_{0}^{s} f(x(u), x(\delta(u))) d u+\int_{0}^{s} g(x(u), x(\delta(u))) d B(u) \\
& +\int_{0}^{s} \int_{|l|<c} H(x(u-), x(\delta(u)-), l) \widetilde{N}(d u, d l) \mid .
\end{aligned}
$$

Therefore

$$
\begin{aligned}
E\left(\sup _{0 \leq s \leq t}|x(s)|^{p}\right) \leq & \frac{1}{(1-\alpha-\beta)^{p}} E\left[\sup _{0 \leq s \leq t} \mid \xi(0)+\int_{0}^{s} f(x(u), x(\delta(u))) d u\right. \\
& \left.+\int_{0}^{s} g(x(u), x(\delta(u))) d B(u)+\left.\int_{0}^{s} \int_{|l|<c} H(x(u-), x(\delta(u)-), l) \tilde{N}(d u, d l)\right|^{p}\right] .
\end{aligned}
$$

Thanks to inequality $|a+b+c+d| \leq 4^{p-1}\left(|a|^{p}+|b|^{p}+|c|^{p}+|d|^{p}\right)$, we deduce that

$$
\begin{aligned}
& E\left(\sup _{0 \leq s \leq t}|x(s)|^{p}\right) \\
& \leq C_{1,1}\left\{E|\xi(0)|^{p}+E\left[\sup _{0 \leq s \leq t}\left|\int_{0}^{s} f(x(u), x(\delta(u))) d u\right|^{p}\right]+E\left[\sup _{0 \leq s \leq t}\left|\int_{0}^{s} g(x(u), x(\delta(u))) d B(u)\right|^{p}\right]\right. \\
& \left.\quad+E\left[\sup _{0 \leq s \leq t}\left|\int_{0}^{s} \int_{|l|<c} H(x(u-), x(\delta(u)-), l) \tilde{N}(d u, d l)\right|^{p}\right]\right\}
\end{aligned}
$$


where $C_{1,1}=\frac{4^{p-1}}{(1-\alpha-\beta)^{p}}$. The Hölder inequality and (H3) imply that

$$
\begin{aligned}
& E\left[\sup _{0 \leq s \leq t}\left|\int_{0}^{s} f(x(u), x(\delta(u))) d u\right|^{p}\right] \\
& \leq T^{p-1} E \int_{0}^{t}|f(x(u), x(\delta(u)))|^{p} d u \\
& \leq T^{p-1} E \int_{0}^{t} K_{3}^{\frac{p}{2}}\left(1+|x(s)|^{2}+|x(\delta(s))|^{2}\right)^{\frac{p}{2}} d s \\
& \leq T^{p-1} E \int_{0}^{t} K_{3}^{\frac{p}{2}}\left(1+2 \sup _{-\tau \leq u \leq s}|x(u)|^{2} \mid\right)^{\frac{p}{2}} d s \\
& \leq 2^{\frac{p-2}{2}} K_{3}^{\frac{p}{2}} T^{p}+2^{p-1} K_{3}^{\frac{p}{2}} T^{p-1} \int_{0}^{t} E\left[\sup _{-\tau \leq u \leq s}|x(u)|^{p}\right] d s .
\end{aligned}
$$

On the other hand, by virtue of the Burkholder-Davis-Gundy inequality (Theorem 1.7.3 in [12]) and (H3), we have

$$
\begin{aligned}
& E\left[\sup _{0 \leq s \leq t}\left|\int_{0}^{s} g(x(u), x(\delta(u))) d B(u)\right|^{p}\right] \\
& \leq\left[\frac{p^{p+1}}{2(p-1)^{p-1}}\right]^{\frac{p}{2}} E\left[\int_{0}^{t}|g(x(u), x(\delta(u)))|^{2} d s\right]^{\frac{p}{2}} \\
& \leq\left[\frac{p^{p+1}}{2(p-1)^{p-1}}\right]^{\frac{p}{2}} T^{\frac{p-2}{2}} E \int_{0}^{t}|g(x(u), x(\delta(u)))|^{p} d s \\
& \quad \leq 2^{\frac{p-2}{2}} K_{3}^{\frac{p}{2}}\left[\frac{p^{p+1}}{2(p-1)^{p-1}}\right]^{\frac{p}{2}} T^{\frac{p}{2}}+2^{p-1} K_{3}^{\frac{p}{2}}\left[\frac{p^{p+1}}{2(p-1)^{p-1}}\right]^{\frac{p}{2}} T^{\frac{p-2}{2}} \int_{0}^{t} E\left[\sup _{-\tau \leq u \leq s}|x(u)|^{p}\right] d s .
\end{aligned}
$$

It follows from (3.1-3.3) and Lemma 4.1 that

$$
E\left(\sup _{0 \leq s \leq t}|x(s)|^{p}\right) \leq C_{1,2}+C_{1,1} E\|\xi\|^{p}+C_{1,3} E\left[\sup _{-\tau \leq u \leq s}|x(u)|^{p}\right] d s,
$$

where $C_{1,2}=C_{1,1}\left[2^{\frac{p-2}{2}} K_{3}^{\frac{p}{2}} T^{p}+2^{\frac{p-2}{2}} K_{3}^{\frac{p}{2}}\left[\frac{p^{p+1}}{2(p-1)^{p-1}}\right]^{\frac{p}{2}} T^{\frac{p}{2}}+C_{p, T}\right], C_{1,3}=C_{1,1}\left[2^{p-1} K_{3}^{\frac{p}{2}} T^{p-1}+2^{p-1}\right.$ $\left.K_{3}^{\frac{p}{2}}\left[\frac{p^{p+1}}{2(p-1)^{p-1}}\right]^{\frac{p}{2}} T^{\frac{p-2}{2}}+C_{p, T}\right]$. Notice that

$$
E\left(\sup _{-\tau \leq s \leq t}|x(s)|^{p}\right) \leq E\|\xi\|^{p} \vee E\left(\sup _{0 \leq s \leq t}|x(s)|^{p}\right),
$$

we get

$$
E\left(\sup _{-\tau \leq s \leq t}|x(s)|^{p}\right) \leq C_{1,2}+C_{1,1} E\|\xi\|^{p}+C_{1,3} \int_{0}^{t} E\left[\sup _{-\tau \leq u \leq s}|x(u)|^{p}\right] d s .
$$


Combining this with the Gronwall's inequality, we get

$$
E\left(\sup _{-\tau \leq s \leq t}|x(s)|^{p}\right) \leq\left[C_{1,2}+C_{1,1} E\|\xi\|^{p}\right] e^{C_{1,3} T} .
$$

This implies our claim immediately by letting

$$
C_{1}=\left[C_{1,2}+C_{1,1} E\|\xi\|^{p}\right] e^{C_{1,3} T}
$$

and the lemma follows.

Lemma 3.3 Under condition (H3), for any $p \geq 2$, there exists a positive constant $C_{2}$, such that

$$
E\left(\sup _{-\tau \leq s \leq T}|y(s)|^{p}\right) \leq C_{2} .
$$

Proof The proof is similar to Lemma 3.2, here we omit it.

For each $n>0$, we define the stopping times

$$
\tau_{n}:=\inf \{t \geq 0:|x(t)| \geq n\}, \quad \sigma_{n}:=\inf \{t \geq 0:|y(t)| \geq n\}
$$

and $v_{n}=\tau_{n} \wedge \sigma_{n}$. (We set inf $\left.\emptyset=\infty\right)$.

Corollary 3.1 Under condition $(\mathrm{H} 2)$, there exists a positive constant $C_{2}(n)$, such that

$$
E\left[\sup _{-\tau \leq s \leq T}\left|y\left(s \wedge v_{n}\right)\right|^{2}\right] \leq C_{2}(n) .
$$

Proof From (H2), for every $n>0$ and $|x| \vee|y| \leq n$, we have

$$
\begin{aligned}
|f(x, y)|^{2} & \leq 2|f(x, y)-f(0,0)|^{2}+2|f(0,0)|^{2} \leq 2 K_{2}(n)\left(|x|^{2}+|y|^{2}\right)+2|f(0,0)|^{2} \\
& \leq K_{4}(n)\left(1+|x|^{2}+|y|^{2}\right) .
\end{aligned}
$$

Similarly, we have

$$
|g(x, y)|^{2} \leq K_{4}(n)\left(1+|x|^{2}+|y|^{2}\right)
$$

and

$$
\int_{|l|<c}|H(x, y, l)|^{\eta} v(d l) \leq K_{4}(n)\left(1+|x|^{\eta}+|y|^{\eta}\right),
$$

where $K_{4}(n)=2^{\eta-1}\left(K_{2}(n) \vee|f(0,0)|^{2} \vee|g(0,0)|^{2} \vee \int_{|l|<c}|H(0,0, l)|^{\eta} v(d l)\right), 2 \leq \eta \leq p$. Thus the corollary follows from Lemma 3.3 .

Lemma 3.4 Under the condition (H2), for any $t \in[0, T]$, we have

$$
E\left|y\left(t \wedge v_{n}\right)-z_{1}\left(t \wedge v_{n}\right)\right|^{2} \leq C_{3}(n) \Delta \text {. }
$$

Proof Clearly, for any $t \in[0, T]$, we may choose a positive integer $k$ such that $t \wedge v_{n} \in[k \Delta,(k+1) \Delta)$ and $k=k(\omega)$ is dependent on the sample path. It follows that

$$
y\left(t \wedge v_{n}\right)-z_{1}\left(t \wedge v_{n}\right)=y\left(t \wedge v_{n}\right)-\bar{y}(k \Delta) .
$$

From Eq. (2.7), we have

$$
\begin{aligned}
& y\left(t \wedge v_{n}\right)-\bar{y}(k \Delta) \\
& \quad \leq \int_{k \Delta}^{t \wedge v_{n}} f\left(z_{1}(s), z_{2}(s)\right) d s+\int_{k \Delta}^{t \wedge v_{n}} g\left(z_{1}(s), z_{2}(s)\right) d B(s)+\int_{k \Delta}^{t \wedge v_{n}} \int_{|l|<c} H\left(z_{1}(s), z_{2}(s), l\right) \widetilde{N}(d s, d l) \\
& \quad+\alpha \sup _{0 \leq s \leq t \wedge v_{n}} y_{1}\left(s, t \wedge v_{n}\right)+\beta \inf _{0 \leq s \leq t \wedge v_{n}} y(s)-\alpha \sup _{0 \leq j \leq k} \bar{y}(j \Delta)-\beta \inf _{0 \leq j \leq k} \bar{y}(j \Delta) .
\end{aligned}
$$


Since $\inf _{0 \leq s \leq t \wedge v_{n}} y(s) \leq \inf _{0 \leq j \leq k} \bar{y}(j \Delta)$, we get from Eq. (3.6) that

$$
\begin{aligned}
& \left|y\left(t \wedge v_{n}\right)-\bar{y}(k \Delta)\right| \\
& \leq\left|\int_{k \Delta}^{t \wedge v_{n}} f\left(z_{1}(s), z_{2}(s)\right) d s+\int_{k \Delta}^{t \wedge v_{n}} g\left(z_{1}(s), z_{2}(s)\right) d B(s)+\int_{k \Delta}^{t \wedge v_{n}} \int_{|l|<c} H\left(z_{1}(s), z_{2}(s), l\right) \widetilde{N}(d s, d l)\right| \\
& \quad+\alpha\left|\sup _{0 \leq s \leq t \wedge v_{n}} y_{1}\left(s, t \wedge v_{n}\right)-\sup _{0 \leq j \leq k} \bar{y}(j \Delta)\right| .
\end{aligned}
$$

Noting that $\sup _{0 \leq s \leq t \wedge v_{n}} y_{1}\left(s, t \wedge v_{n}\right)=\sup _{1 \leq j \leq k} \bar{y}(j \Delta) \vee \sup _{k \Delta \leq s \leq t \wedge v_{n}} y(s)$, we derive

$$
\left|\sup _{0 \leq s \leq t \wedge v_{n}} y_{1}\left(s, t \wedge v_{n}\right)-\sup _{0 \leq j \leq k} \bar{y}(j \Delta)\right| \leq \sup _{k \Delta \leq s \leq t \wedge v_{n}}|y(s)-\bar{y}(k \Delta)| .
$$

In fact, if $\sup _{0 \leq s \leq t \wedge v_{n}} y_{1}\left(s, t \wedge v_{n}\right)=\sup _{1 \leq j \leq k} \bar{y}(j \Delta)$, (3.7) obviously holds, and if

$$
\sup _{0 \leq s \leq t \wedge v_{n}} y_{1}\left(s, t \wedge v_{n}\right)=\sup _{k \Delta \leq s \leq t \wedge v_{n}} y(s),
$$

we have

$$
\sup _{0 \leq s \leq t \wedge v_{n}} y_{1}\left(s, t \wedge v_{n}\right)-\sup _{0 \leq j \leq k} \bar{y}(j \Delta) \leq \sup _{k \Delta \leq s \leq t \wedge v_{n}} y(s)-\bar{y}(k \Delta) \leq \sup _{k \Delta \leq s \leq t \wedge v_{n}}|y(s)-\bar{y}(k \Delta)| .
$$

Thus, we see that

$$
\begin{aligned}
\sup _{k \Delta \leq s \leq t \wedge v_{n}}|y(s)-\bar{y}(k \Delta)| \leq & \sup _{k \Delta \leq s \leq t \wedge v_{n}} \mid \int_{k \Delta}^{s} f\left(z_{1}(u), z_{2}(u)\right) d u+\int_{k \Delta}^{s} g\left(z_{1}(u), z_{2}(u)\right) d B(u) \\
& +\int_{k \Delta|l|<c}^{s} \int_{k \Delta} H\left(z_{1}(u), z_{2}(u), l\right) \tilde{N}(d u, d l)\left|+\alpha \sup _{k \Delta \leq s \leq t \wedge v_{n}}\right| y(s)-\bar{y}(k \Delta) \mid .
\end{aligned}
$$

This implies that

$$
\begin{aligned}
\sup _{k \Delta \leq s \leq t \wedge v_{n}}|y(s)-\bar{y}(k \Delta)| \leq & \sup _{k \Delta \leq s \leq t \wedge v_{n}} \frac{1}{1-\alpha} \mid \int_{k \Delta}^{s} f\left(z_{1}(u), z_{2}(u)\right) d u+\int_{k \Delta}^{s} g\left(z_{1}(u), z_{2}(u)\right) d B(u) \\
& +\int_{k \Delta|l|<c}^{s} \int_{k} H\left(z_{1}(u), z_{2}(u), l\right) \tilde{N}(d u, d l) \mid .
\end{aligned}
$$

Combining this with inequality $|a+b+c|^{2} \leq 3\left(|a|^{2}+|b|^{2}+|c|^{2}\right)$, one can get

$$
\begin{aligned}
& E\left[\sup _{k \Delta \leq s \leq t \wedge v_{n}}|y(s)-\bar{y}(k \Delta)|^{2}\right] \\
& \leq 3 C_{3,1}\left\{E\left[\sup _{k \Delta \leq s \leq t \wedge v_{n}}\left|\int_{k \Delta}^{s} f\left(z_{1}(u), z_{2}(u)\right) d u\right|^{2}\right]+E\left[\sup _{k \Delta \leq s \leq t \wedge v_{n}}\left|\int_{k \Delta}^{s} g\left(z_{1}(u), z_{2}(u)\right) d B(u)\right|^{2}\right]\right. \\
& \left.+E\left[\sup _{k \Delta \leq s \leq t \wedge v_{n}}\left|\int_{k \Delta}^{s} \int_{|l|<c} H\left(z_{1}(u), z_{2}(u), l\right) \widetilde{N}(d u, d l)\right|^{2}\right]\right\}
\end{aligned}
$$


where $C_{3,1}=\frac{1}{(1-\alpha)^{2}}$. Using Doob's martingale inequality, Itô's isometry property and Eq. (3.5) yields that

$$
\begin{aligned}
& E\left[\sup _{k \Delta \leq s \leq t \wedge v_{n}}\left|\int_{|k \Delta| l \mid<c}^{s} \int_{t \wedge c} H\left(z_{1}(s), z_{2}(s), l\right) \tilde{N}(d u, d l)\right|^{2}\right] \\
& \leq 4 E \int_{k \Delta}^{t \wedge v_{n}} \int_{|l|<c}^{t \wedge v_{n}}\left|H\left(z_{1}(u), z_{2}(u), l\right)\right|^{2} d s v(d l) \\
& \leq 4 K_{4}(n) \int_{k \Delta}^{t \wedge}\left(1+2 E\left[\sup _{-\tau \leq u \leq s}|y(u)|^{2}\right]\right) d s .
\end{aligned}
$$

Substituting Eqs. (3.2) and (3.3) with $p=2, K_{3}=K_{4}(n)$ and Eq. (3.9) into (3.8), we get

$$
\begin{aligned}
& E\left[\sup _{k \Delta \leq s \leq t \wedge v_{n}}|y(s)-\bar{y}(k \Delta)|^{2}\right] \\
& \quad \leq 3 C_{3,1} K_{4}(n)(T+8) \int_{k \Delta}^{t \wedge v_{n}}\left(1+2 E\left[\sup _{-\tau \leq u \leq s}|y(u)|^{2}\right]\right) d s .
\end{aligned}
$$

It follows from Eq. (3.10) and Corollary 3.1 that

$$
\begin{aligned}
E\left[\sup _{k \Delta \leq s \leq t \wedge v_{n}}|y(s)-\bar{y}(k \Delta)|^{2}\right] & \leq 3 C_{3,1} K_{4}(n)(T+8) \int_{k \Delta}^{t \wedge v_{n}}\left(1+2 C_{2}(n)\right) d s \\
& \leq 3 C_{3,1} K_{4}(n)(T+8)\left(1+2 C_{2}(n)\right) \Delta .
\end{aligned}
$$

Therefore,

$$
E\left|y\left(t \wedge v_{n}\right)-z_{1}\left(t \wedge v_{n}\right)\right|^{2}=E\left|y\left(t \wedge v_{n}\right)-\bar{y}(k \Delta)\right|^{2} \leq E \sup _{k \Delta \leq s \leq t}\left|y\left(s \wedge v_{n}\right)-\bar{y}(k \Delta)\right|^{2} \leq C_{3}(n) \Delta(3.11)
$$

where $C_{3}(n)=3 C_{3,1} K_{4}(n)(T+8)\left(1+2 C_{2}(n)\right)$. This completes the proof.

Corollary 3.2 Under condition (H2), we have

$$
E \sup _{0 \leq t \leq T}\left|y(t)-z_{1}(t)\right|^{2} \leq C_{3}(n) \Delta .
$$

Proof For any $t \in[0, T]$, we can choose a positive integer $k$ such that $t \in[k \Delta,(k+1) \Delta)$ and $k=k(\omega)$ is dependent on the sample path. Therefore

$$
y(t)-z_{1}(t)=y(t)-\bar{y}(k \Delta)
$$

By Eq. (3.11), we have

$$
E \sup _{k \Delta \leq t<(k+1) \Delta}\left|y(t)-z_{1}(t)\right|^{2}=E \sup _{k \Delta \leq t<(k+1) \Delta}|y(t)-\bar{y}(k \Delta)|^{2} \leq C_{3}(n) \Delta,
$$

where $C_{3}(n)$ is independent of $k$. Thus, for any $k$, we can get $E \sup _{k \Delta \leq t<(k+1) \Delta}\left|y(t)-z_{1}(t)\right|^{2} \leq C_{3}(n) \Delta$ and the Corollary follows.

Lemma 3.5 Under $(\mathrm{H} 1)$ and $(\mathrm{H} 2)$, if $\Delta$ is small enough such that $(\rho+1) \Delta \leq 1$, then there exists a positive constant $C_{4}(n)$, such that

$$
E\left|y\left(\delta\left(t \wedge v_{n}\right)\right)-z_{2}\left(t \wedge v_{n}\right)\right|^{2} \leq C_{4}(n) \Delta, \quad \forall t \in[0, T] .
$$

Proof One just needs to repeat the proof of the Lemma 3.3 as in Mao [13], so we omit the detailed proof. 


\section{Main result}

The primary aim of this paper is to establish the following main result.

Theorem 4.1 Under hypotheses (H1-H3), the approximate solution (2.6) converges to the true solution of Eq. (1.1) in the sense

$$
\lim _{\Delta \rightarrow 0} E\left[\sup _{0 \leq t \leq T}|x(t)-y(t)|^{2}\right]=0
$$

Proof It is obvious that

$$
\begin{aligned}
& E \sup _{0 \leq t \leq T}|x(t)-y(t)|^{2} \\
& \left.\left.\leq E\left[\sup _{0 \leq t \leq T}|x(t)-y(t)|^{2} I_{\left\{\tau_{n}>T\right.} \text { and } \sigma_{n}>T\right\}\right]+E\left[\sup _{0 \leq t \leq T}|x(t)-y(t)|^{2} I_{\left\{\tau_{n} \leq T\right.} \text { or } \sigma_{n} \leq T\right\}\right] .
\end{aligned}
$$

By the Young inequality

$$
a b \leq 2 h \frac{a^{\frac{p}{2}}}{p}+\frac{p-2}{p h^{\frac{2}{p-2}}} b^{\frac{p}{p-2}},
$$

where $a, b, h>0$ and $p>2$, we obtain

$$
\left.\left.E\left[\sup _{0 \leq t \leq T}|x(t)-y(t)|^{2} I_{\left\{\tau_{n} \leq T\right.} \text { or } \sigma_{n} \leq T\right\}\right] \leq E\left[\frac{2 h}{p} \sup _{0 \leq t \leq T}|x(t)-y(t)|^{p}\right]+E\left[\frac{p-2}{p} \frac{1}{h^{\frac{2}{p-2}}} I_{\left\{\tau_{n} \leq T\right.} \text { or } \sigma_{n} \leq T\right\}\right] .
$$

Consequently,

$$
\left.E\left[\sup _{0 \leq t \leq T}|x(t)-y(t)|^{2} I_{\left\{\tau_{n} \leq T\right.} \text { or } \sigma_{n} \leq T\right\}\right] \leq \frac{2 h}{p} E\left[\sup _{0 \leq t \leq T}|x(t)-y(t)|^{p}\right]+\frac{p-2}{p} \frac{1}{h^{\frac{2}{p-2}}} P\left(\tau_{n} \leq T \text { or } \sigma_{n} \leq T\right) .
$$

Set $C_{5}=C_{1} \vee C_{2}$. Notice that

$$
P\left(\tau_{n} \leq T\right) \leq E\left[I_{\left\{\tau_{n} \leq T\right\}} \frac{\left|x\left(\tau_{n}\right)\right|^{p}}{n^{p}}\right] \leq \frac{1}{n^{p}} E\left[\sup _{-\tau \leq t \leq T}|x(t)|^{p}\right] \leq \frac{C_{5}}{n^{p}}
$$

and

$$
P\left(\sigma_{n} \leq T\right) \leq \frac{C_{5}}{n^{p}}
$$

We get

$$
P\left(\tau_{n} \leq T \text { or } \sigma_{n} \leq T\right) \leq P\left(\tau_{n} \leq T\right)+P\left(\sigma_{n} \leq T\right) \leq \frac{2 C_{5}}{n^{p}}
$$

Moreover,

$$
E\left[\sup _{0 \leq t \leq T}|x(t)-y(t)|^{p}\right] \leq 2^{p-1} E\left[\sup _{0 \leq t \leq T}|x(t)|^{p}+\sup _{0 \leq t \leq T}|y(t)|^{p}\right] \leq 2^{p} C_{5} .
$$

Substituting these inequalities above into Eq. (4.1) leads to

$$
E\left[\sup _{0 \leq t \leq T}|x(t)-y(t)|^{2}\right] \leq E\left[\sup _{0 \leq t \leq T}|x(t)-y(t)|^{2} I_{\left\{\tau_{n}>T \text { and } \sigma_{n}>T\right\}}\right]+\frac{2^{p+1} h C_{5}}{p}+\frac{2(p-2) C_{5}}{p h^{\frac{2}{p-2}} n^{p}}
$$


Let us now estimate the first term on the right-hand side of Eq. (4.2). Clearly,

$$
\begin{aligned}
& E\left[\sup _{0 \leq t \leq T}|x(t)-y(t)|^{2} I_{\left\{\tau_{n}>T \text { and } \sigma_{n}>T\right\}}\right] \\
& =E\left[\sup _{0 \leq t \leq T}|x(t)-y(t)|^{2} 1_{\left\{v_{n}>T\right\}}\right] \leq E\left[\sup _{0 \leq t \leq T}\left|x\left(t \wedge v_{n}\right)-y\left(t \wedge v_{n}\right)\right|^{2}\right] .
\end{aligned}
$$

Thanks to Eqs. (1.1) and (2.6), we derive

$$
\begin{aligned}
& \left|x\left(t \wedge v_{n}\right)-y\left(t \wedge v_{n}\right)\right| \\
& \leq \mid \int_{0}^{t \wedge v_{n}}\left[f(x(s), x(\delta(s)))-f\left(z_{1}(s), z_{2}(s)\right)\right] d s+\int_{0}^{t \wedge v_{n}}\left[g(x(s), x(\delta(s)))-g\left(z_{1}(s), z_{2}(s)\right)\right] d B(s) \\
& \quad+\int_{0}^{t \wedge v_{n}} \int_{|l|<c}\left[H(x(s-), x(\delta(s)-), l)-H\left(z_{1}(s), z_{2}(s), l\right)\right] \widetilde{N}(d s, d l)|+\beta| \inf _{0 \leq s \leq t \wedge v_{n}} x(s)-y(s) \mid \\
& \quad+\beta\left|y(s)-\inf _{0 \leq s \leq t \wedge v_{n}} y_{1}\left(s, t \wedge v_{n}\right)\right|+\alpha\left|\sup _{0 \leq s \leq t \wedge v_{n}} x(s)-\sup _{0 \leq s \leq t \wedge v_{n}} y_{1}\left(s, t \wedge v_{n}\right)\right|,
\end{aligned}
$$

for $0 \leq t \wedge v_{n} \leq T$. Obviously,

$$
\left|\inf _{0 \leq s \leq t \wedge v_{n}} x(s)-\inf _{0 \leq s \leq t \wedge v_{n}} y(s)\right| \leq \sup _{0 \leq s \leq t \wedge v_{n}}|x(s)-y(s)| .
$$

By the definition of $y_{1}(t)$, we have

$$
\left|y(s)-\inf _{0 \leq s \leq t \wedge v_{n}} y_{1}\left(s, t \wedge v_{n}\right)\right| \leq\left|y(s)-y_{1}\left(s, t \wedge v_{n}\right)\right|=\left|y(s)-z_{1}(s)\right| I_{\left[0, I_{\Delta}\left[t \wedge v_{n} \wedge T\right]\right]}(s) .
$$

Noting that $\sup _{0 \leq s \leq t \wedge v_{n}} y_{1}\left(s, t \wedge v_{n}\right) \leq \sup _{0 \leq s \leq t \wedge v_{n}} y(s)$, we can get

$$
\begin{aligned}
\sup _{0 \leq s \leq t \wedge v_{n}} y_{1}\left(s, t \wedge v_{n}\right)-\sup _{0 \leq s \leq t \wedge v_{n}} x(s) & \leq \sup _{0 \leq s \leq t \wedge v_{n}} y(s)-\sup _{0 \leq s \leq t \wedge v_{n}} x(s) \\
& \leq\left|\sup _{0 \leq s \leq t \wedge v_{n}} x(s)-\sup _{0 \leq s \leq t \wedge v_{n}} y(s)\right| \\
& \leq \sup _{0 \leq s \leq t \wedge v_{n}}|x(s)-y(s)|,
\end{aligned}
$$

which implies

$$
\left|\sup _{0 \leq s \leq t \wedge v_{n}} x(s)-\sup _{0 \leq s \leq t \wedge v_{n}} y_{1}\left(s, t \wedge v_{n}\right)\right| \leq \sup _{0 \leq s \leq t \wedge v_{n}}|x(s)-y(s)| .
$$

Substituting Eqs. (4.5), (4.6) and (4.7) into Eq. (4.4) gives

$$
\begin{aligned}
& \left|x\left(s \wedge v_{n}\right)-y\left(s \wedge v_{n}\right)\right| \\
& \leq \mid \int_{0}^{s \wedge v_{n}}\left[f(x(u), x(\delta(u)))-f\left(z_{1}(u), z_{2}(u)\right)\right] d u+\int_{0}^{s \wedge v_{n}}\left[g(x(u), x(\delta(u)))-g\left(z_{1}(u), z_{2}(u)\right)\right] d B(u) \\
& \left.\quad+\int_{0}^{s \wedge v_{n}} \int_{|l|<c}\left[H(x(u-), x(\delta(u)-), l)-H\left(z_{1}(u), z_{2}(u), l\right)\right] \widetilde{N}(d u, d l)\right\} \mid \\
& \quad+(\alpha+\beta) \sup _{0 \leq s \leq t \wedge v_{n}}|x(s)-y(s)|+\left|y(s)-z_{1}(s)\right| I_{\left[0, I_{\Delta}\left[t \wedge v_{n} \wedge T\right]\right]}(s) .
\end{aligned}
$$


This proves that

$$
\begin{aligned}
& \sup _{0 \leq s \leq t \wedge v_{n}}\left|x\left(s \wedge v_{n}\right)-y\left(s \wedge v_{n}\right)\right| \\
& \leq \sup _{0 \leq s \leq t \wedge v_{n}} \mid \int_{0}^{s}\left[f(x(u), x(\delta(u)))-f\left(z_{1}(u), z_{2}(u)\right)\right] d u+\int_{0}^{s}\left[g(x(u), x(\delta(u)))-g\left(z_{1}(u), z_{2}(u)\right)\right] d B(u) \\
& \left.\quad+\int_{0}^{s} \int_{|l|<c}\left[H(x(u-), x(\delta(u)-), l)-H\left(z_{1}(u), z_{2}(u), l\right)\right] \tilde{N}(d u, d l)\right\} \mid \\
& \quad+(\alpha+\beta) \sup _{0 \leq s \leq t \wedge v_{n}}|x(s)-y(s)|+\sup _{0 \leq s \leq T}\left|y(s)-z_{1}(s)\right| .
\end{aligned}
$$

\section{Consequently,}

$$
\begin{aligned}
\sup _{0 \leq s \leq t \wedge v_{n}}|x(s)-y(s)| & \\
\leq & \frac{1}{1-\alpha-\beta} \sup _{0 \leq s \leq t \wedge v_{n}} \mid \int_{0}^{s}\left[f(x(u), x(\delta(u)))-f\left(z_{1}(u), z_{2}(u)\right)\right] d u \\
& +\int_{0}^{s}\left[g(x(u), x(\delta(u)))-g\left(z_{1}(u), z_{2}(u)\right)\right] d B(u) \\
& +\int_{0}^{s} \int_{|l|<c}\left[H(x(u-), x(\delta(u)-), l)-H\left(z_{1}(u), z_{2}(u), l\right)\right] \widetilde{N}(d u, d l)\left|+\frac{1}{1-\alpha-\beta} \sup _{0 \leq s \leq T}\right| y(s)-z_{1}(s) \mid .
\end{aligned}
$$

Using the Doob martingale inequality, the local Lipschitz condition (H2) and Corollary 3.2 gives

$$
\begin{aligned}
E & {\left[\sup _{0 \leq s \leq t \wedge v_{n}}|x(s)-y(s)|^{2}\right] } \\
\leq & \frac{3}{1-\alpha-\beta}\left\{E \sup _{0 \leq s \leq t \wedge v_{n}}\left|\int_{0}^{s}\left[f(x(u), x(\delta(u)))-f\left(z_{1}(u), z_{2}(u)\right)\right] d u\right|^{2}\right. \\
& +E \sup _{0 \leq s \leq t \wedge v_{n}}\left|\int_{0}^{s}\left[g(x(u), x(\delta(u)))-g\left(z_{1}(u), z_{2}(u)\right)\right] d B(u)\right|^{2} \\
& \left.+E \sup _{0 \leq s \leq t \wedge v_{n}}\left|\int_{0}^{s} \int_{|l|<c}^{s}\left[H(x(u-), x(\delta(u)-), l)-H\left(z_{1}(u), z_{2}(u), l\right)\right] \tilde{N}(d u, d l)\right|^{2}\right\}+\frac{C_{3}(n)}{1-\alpha-\beta} \Delta \\
\leq & \frac{3 K_{2}(n)(T+4)}{1-\alpha-\beta} E\left[\int_{0}^{t \wedge v_{n}}\left(\left|x(s)-z_{1}(s)\right|^{2}+\left|x(\delta(s))-z_{2}(s)\right|^{2}\right) d s\right] \\
& +\frac{12 K_{2}(n)}{1-\alpha-\beta} E\left[\int_{0}^{t \wedge v_{n}}\left(\left|x(s-)-z_{1}(s)\right|^{2}+\left|x(\delta(s)-)-z_{2}(s)\right|^{2}\right) d s\right]+\frac{C_{3}(n)}{1-\alpha-\beta} \Delta .
\end{aligned}
$$




\section{It follows that}

$$
\begin{aligned}
& E\left[\sup _{0 \leq s \leq t \wedge v_{n}}|x(s)-y(s)|^{2}\right] \leq \frac{6 K_{2}(n)(T+4)}{1-\alpha-\beta} \\
& \quad \times E\left[\int_{0}^{t \wedge v_{n}}\left(|x(s)-y(s)|^{2}+\left|y(s)-z_{1}(s)\right|^{2}+|x(\delta(s))-y(\delta(s))|^{2}+\left|y(\delta(s))-z_{2}(s)\right|^{2}\right) d s\right] \\
& \quad+\frac{24 K_{2}(n)}{1-\alpha-\beta} E\left[\int_{0}^{t \wedge v_{n}}\left(|x(s-)-y(s)|^{2}+\left|y(s)-z_{1}(s)\right|^{2}+|x(\delta(s)-)-y(\delta(s))|^{2}+\left|y(\delta(s))-z_{2}(s)\right|^{2}\right) d s\right] \\
& \quad+\frac{C_{3}(n)}{1-\alpha-\beta} \Delta .
\end{aligned}
$$

Note that $y(t)=\xi(t)$ for any $t \in[-\tau, 0]$ and

$$
|x(s-)-y(s)|^{2}+|x(\delta(s)-)-y(\delta(s))|^{2} \leq 2 \sup _{0 \leq u \leq s}|x(u)-y(u)|^{2} .
$$

We get

$$
\begin{aligned}
& E\left[\sup _{0 \leq s \leq t \wedge v_{n}}|x(s)-y(s)|^{2}\right] \\
& \leq \frac{6 K_{2}(n)(T+8)}{1-\alpha-\beta}\left\{2 E\left[\int_{0}^{t \wedge v_{n}}\left(\sup _{0 \leq u \leq s}|x(u)-y(u)|^{2}\right) d s\right]+E\left[\int_{0}^{t \wedge v_{n}}\left(\left|y(s)-z_{1}(s)\right|^{2}+\left|y(\delta(s))-z_{2}(s)\right|^{2}\right) d s\right]\right\} \\
& \quad+\frac{C_{3}(n)}{1-\alpha-\beta} \Delta .
\end{aligned}
$$

Combining this with Lemmas 3.4 and 3.5, we see that

$$
\begin{aligned}
& E\left[\sup _{0 \leq s \leq t \wedge v_{n}}|x(s)-y(s)|^{2}\right] \\
& \quad \leq C_{5,1}(n) \int_{0}^{t \wedge v_{n}} E\left[\sup _{0 \leq u \leq s}|x(u)-y(u)|^{2}\right] d s+C_{5,2}(n) \Delta,
\end{aligned}
$$

where $C_{5,1}(n)=\frac{12 K_{2}(n)(T+8)}{1-\alpha-\beta}, C_{5,2}(n)=\frac{6 K_{2}(n)(T+8)}{1-\alpha-\beta}\left(C_{3}(n)+C_{4}(n)\right) T+\frac{C_{3}(n)}{1-\alpha-\beta}$. Then by the Gronwall inequality, we derive

$$
E\left[\sup _{0 \leq s \leq t \wedge v_{n}}|x(s)-y(s)|^{2}\right] \leq C_{5,2}(n) e^{C_{5,1}(n) T} \Delta .
$$

Thus, by Eqs. (4.2), (4.3) and (4.8) we get

$$
E\left[\sup _{0 \leq t \leq T}|x(t)-y(t)|^{2}\right] \leq C_{5,2}(n) e^{C_{5,1}(n) T} \Delta+\frac{2^{p+1} h C_{5}}{p}+\frac{2(p-2) C_{5}}{p h^{\frac{2}{p-2}} n^{p}} .
$$

Now, for any $\epsilon>0$, we can choose a sufficiently small $h$ such that $\frac{2^{p+1} h C_{5}}{p}<\frac{\epsilon}{3}$, then choose $n$ so large that $\frac{2(p-2) C_{5}}{p h^{\frac{2}{p-2}} n^{p}}<\frac{\epsilon}{3}$ and finally choose $\Delta$ sufficiently small such that $C_{5,2}(n) e^{C_{5,1}(n) T} \Delta<\frac{\epsilon}{3}$. So

$$
E\left[\sup _{0 \leq t \leq T}|x(t)-y(t)|^{2}\right] \leq \epsilon
$$

This completes the proof. 
Theorem 4.1 shows that under conditions $(\mathrm{H} 1-\mathrm{H} 3)$ the Euler-Maruyama approximate solutions strongly converge to the true solutions. However, Theorem 4.1 does not give the order of the convergence. In the following, we will reveal the order of convergence, here we need the global Lipschitz condition instead of the local Lipschitz condition as follows.

(H4) For $2 \leq \eta \leq p$, there exists a positive constant $K_{2}$, such that

$$
\left|f\left(x_{1}, y_{1}\right)-f\left(x_{2}, y_{2}\right)\right|^{2} \vee\left|g\left(x_{1}, y_{1}\right)-g\left(x_{2}, y_{2}\right)\right|^{2} \leq K_{2}\left(\left|x_{1}-x_{2}\right|^{2}+\left|y_{1}-y_{2}\right|^{2}\right)
$$

and

$$
\int_{|l|<c}\left|H\left(x_{1}, y_{1}, l\right)-H\left(x_{2}, y_{2}, l\right)\right|^{\eta} v(d l) \leq K_{2}\left(\left|x_{1}-x_{2}\right|^{\eta}+\left|y_{1}-y_{2}\right|^{\eta}\right),
$$

for $x_{k}, y_{k} \in R^{d}, k=1,2$.

The global Lipschitz condition (H4) implies the linear growth condition

$$
|f(x, y)|^{2} \vee|g(x, y)|^{2} \leq K_{4}\left(1+|x|^{2}+|y|^{2}\right)
$$

and

$$
\int_{|l|<c}|H(x, y, l)|^{\eta} v(d l) \leq K_{4}\left(1+|x|^{\eta}+|y|^{\eta}\right),
$$

where $K_{4}=2^{\eta-1}\left(K_{2} \vee|f(0,0)|^{2} \vee|g(0,0)|^{2} \vee \int_{|l|<c}|H(0,0, l)|^{\eta} v(d l)\right), 2 \leq \eta \leq p$.

By Theorem 4.1, we get

Corollary 4.1 Under hypothesis (H1) and the global Lipschitz condition (H4), we have

$$
E\left[\sup _{0 \leq t \leq T}|x(t)-y(t)|^{2}\right] \leq C_{6} \Delta
$$

where $C_{6}$ is a positive constant independent of $\Delta$.

Proof The proof is followed from the Corollary 5.1 in Mao [13] and is omitted here.

\section{Conclusion and outlook}

In this paper, the numerical solutions of doubly perturbed stochastic delay differential equations driven by Lèvy process are considered. We define the numerical solutions and show that the numerical solutions converge to the true solutions under the local Lipschitz condition. Moreover, we give the order of convergence under the global Lipschitz condition. In our future works, we will investigate doubly perturbed stochastic delay differential equations with Markovian switching and doubly perturbed neutral SDDEs.

Acknowledgments The authors would like to thank two anonymous referees for their helpful comments and suggestions which greatly improved this paper. This paper was partially supported by the National Science Foundation of P. R. China (10871041).

Open Access This article is distributed under the terms of the Creative Commons Attribution License which permits any use, distribution, and reproduction in any medium, provided the original author(s) and the source are credited. 


\section{References}

1. Applebaum, D.; Siakalli, M.: Asymptotic stability of stochastic differential equations driven by Lévy noise. J. Appl. Probab. 46, 1116-1129 (2009)

2. Bo, L.; Zhang, T.: Large deviations for perturbed reflected diffusion processes. Stoch. Int. J. Probab. Stoch. Process. 81, 531-543 (2009)

3. Buckwar, E.: Introduction to the numerical analysis of stochastic delay differential equations. J. Comput. Appl. Math. 125, 297-307 (2000)

4. Chaumont, L.; Doney, R.A.; Hu, Y.: Upper and lower limits of doubly perturbed Brownian motion. Ann. Inst. H Poincaré Probab. Statist. 36, 219-249 (2000)

5. Davis, B.: Weak limits of perturbed random walks and the equation $Y_{t}=B(t)+\alpha \sup _{s \leq t} Y_{s}+\beta \sup _{s \leq t} Y_{s}$. Ann. Probab. 24, 2007-2023 (1996)

6. Doney, R.A.; Zhang, T.: Perturbed Skorohod equations and perturbed reflected diffusion processes. Ann. Inst. H Poincaré Probab. Statist. 41, 107-121 (2005)

7. Higham, D.J.; Mao, X.; Stuart, A.M.: Strong convergence of numerical methods for nonlinear stochastic differential equations. SIAM J. Numer. Anal. 40, 1041-1063 (2002)

8. Küchler, U.; Platen, E.: Strong discrete time approximation of stochastic differential equations with time delay. Math. Comput. Simul. 54, 189-205 (2000)

9. Kunita, H.: Stochastic differential equations based on Lèvy processes and stochastic flows of diffeomorphisms. In: Rao M.M. (ed.). Real and Stochastic Analysis, New Perspectives, pp. 305-375. Birkhaüser, Boston (2004)

10. Li, R.; Chang, Z.: Convergence of numerical solution to stochastic delay differential equation with Poisson jump and Markovian switching. Appl. Math. Comput. 184, 451-463 (2007)

11. Luo, J.: Doubly perturbed jump-diffusion processes. J. Math. Anal. Appl. 351, 147-151 (2009)

12. Mao, X.: Stochastic Differential Equations and Applications, 2nd edn. Horwood Publications, Auckland (2008)

13. Mao, X., Sabanis, S.: Numerical solutions of stochastic differential delay equations under local Lipschitz condition. J. Comput. Appl. Math. 151, 215-227 (2003)

14. Norris, J.R.; Rogers, L.C.G.; Williams, D.: Self-avoiding random walk: a Brownian motion model with local time drift. Probab. Theory Relat. Fields 74, 271-287 (1987)

15. Perman, M.; Werner, W.: Perturbed Brownian motions. Probab. Theory Relat. Fields 108, 357-383 (1997)

16. Saito, Y.; Mitsui, T.: Stability analysis of numerical schemes for stochastic differential equations. SIAM J. Numer. Anal. 33, 2254-2267 (1996)

17. Tóth, B.: Generalized Ray-Knight theory and limit theorems for self-interacting random walks on $Z^{1}$. Ann. Probab. 24, 13241367 (1996)

18. Tóth, B.: The true self-avoiding walk with bond repulsion on Z: limit theorems. Ann. Probab. 23, 1523-1556 (1996)

19. Wang, X.; Gan, S.: Compensated stochastic theta methods for stochastic differential equations with jumps. Appl. Numer. Math. 60, 877-887 (2010) 